\title{
SURVEY OF THE PROBLEMS AND SOLUTIONS OF ARRAYED WAVEGUIDE GRATINGS USED IN THE OPTICAL NETWORKS
}

\author{
Abdulazizjon ABDULLAEV*, Ján TURÁN** \\ * Department of Telecommunication Engineering, Fergana Branch of the Tashkent University of Information Technologies, 185, \\ Mustakillik, Fergana town, Uzbekistan, tel. 99873 226-82-33; mob.tel.+99 890- 303-35-11, e-mail: abdulaziz.telecom@ gmail.com \\ ** Department of Electronics and Multimedia Telecommunications, Faculty of Electrical Engineering and Informatics, Technical \\ University of Košice, Park Komenského 13, 04001 Košice, Slovak Republic, tel. +55 602 2943, e-mail: jan.turan@ @uke.sk
}

\begin{abstract}
Arrayed waveguide grating $(A W G)$ is very attractive device for the all optical networks. This paper is devoted to the principle of $A W G$, the advantages and drawbacks of $A W G$. The problems of $A W G$ were described and the decisions of these problems are resulted as well.
\end{abstract}

Keywords: Arrayed waveguide grating (AWG), optical fiber, optical multiplexer and demultiplexer, wavelength converter.

\section{INTRODUCTION}

AWG is passive optical device used in high speed optical networks, for instant, in the Dense Wavelength Division Multiplexing (DWDM) networks [1], [2], [3]. This device is capable of multiplexing a large number of wavelengths into a single optical fiber, thereby increasing the transmission capacity of optical networks considerably. AWG is based on the principles of diffractions and this device is sometimes called as optical waveguide, the waveguide grating routers, the phase array, or phasar [4], [5]. It can be used as a $\mathrm{Nx} 1$ multiplexer and $1 \times \mathrm{N}$ demultiplexer, add/drop multiplexer/demultiplexer and filter, router and $\mathrm{NxN}$ optical switch [1], [2], [3].

AWG generally consists of $\mathrm{N}$ input waveguides, $\mathrm{N}$ output waveguides, two focusing slab regions (coupler) that are also called free propagation region (FPR), and an array of waveguide gratings that is made of $\mathrm{M}$ uncoupled waveguides (Fig.1). The AWG is a generalization of the Mach Zehnder interferometer: In AWG several copies of the same signal, but shifted in phase by different amount are added together. The beam what is propagated in input waveguides enters the slab region where it becomes divided. At the input ends of waveguide array the beam is coupled into the arrayed waveguides and then propagates through each individual arrayed waveguides to the output. The lengths of the arrayed waveguides are so the optical path length difference between adjacent waveguides equals an integer multiple of the center wavelength of the demultiplexer. For this center wavelength, the fields in individual waveguides will get to the output aperture with equal phase. So, the arrayed waveguides are used as the filters. Each of them lets to achieve only one wavelength to the output despite all wavelengths enter to the each waveguide.

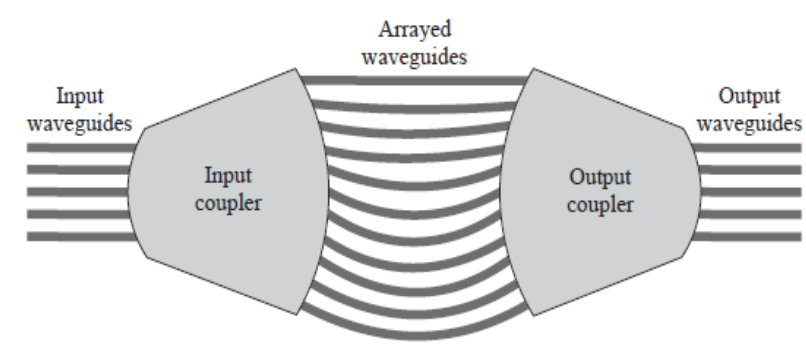

Fig. 1 Schematic configuration of AWG

The transfer function between the central input port of an AWG demultiplexer and its output port located at distance $y_{q o}$ from its central output port is given by [6]

$$
H_{q}(f)=\sum_{m=-p}^{p} C_{m} \exp \left[2 \pi f t_{m}-j k f y_{m} y_{q 0}\right]
$$

where $f$ is the frequency of the optical spectrum and $C_{m}$ the optical power of the $m^{\text {th }}$ grating waveguide normalized to the total power $\left(C_{m}\right.$ is a dimensionless quantity), located at position $y_{m}$ from the central grating waveguide.

Tunable transmitters and tunable receivers need to be attached respectively at the input and output ports of AWG to be able to send and receive on all possible wavelengths.

The main materials of AWG are: silica-on silica, Indium-Phosphide ( $\mathrm{InP})$, Lithium niobate $\left(\mathrm{LiNbO}_{3}\right), \mathrm{Ge}$ doped silica $\left(\mathrm{GeO}_{2}(\mathrm{x})+\mathrm{SiO}_{2}(1-\mathrm{x})\right), \quad \mathrm{SiO}_{2}-\mathrm{Ta}_{2} \mathrm{O}_{3}$, PolyMethyl-Metha Acrylate (PMMA) based AWG devices [7]. 
The AWG has following advantages: suitable for large - scale integration, more fabrication tolerance, compatible with conventional waveguide technologies and better for mass production.

In the paper problems of AWG were described and the decisions of these problems are resulted as well.

\section{DEPENDENCE OF THE WAVELENGTH ON THE TEMPERATURE}

In passive devices (as $\mathrm{AWG}$ ) temperature dependence factor is very important because no power supply for active temperature control is available. The AWG central wavelength changes with temperature. Active temperature stabilization by a heater or Peltier cooler is often used, but it requires continuous power consumption of several watts and temperature control electronics. This can be avoided with an athermal design, with substantially reduced temperature sensitivity. Dependence of wavelength on the temperature in the different AWG-materials was investigated in [7].

\section{CHROMATIC DISPERSION}

Chromatic dispersion is the term given to the phenomenon by which different spectral components of a pulse travel at different velocities [8]. In other words, because of chromatic dispersion, packets that are transmitted on different wavelengths experience different propagation delays. The use of dispersion-compensating fiber alleviates the effects of chromatic dispersion.

Chromatic dispersion follows from the fact the color of the light has an effect on its propagation speed. Because of chromatic dispersion the propagation delay in different channels is different. A typical AWG has a symmetric intensity distribution across the waveguide array, and as such its chromatic dispersion D is negligible. However in a practical AWG this symmetry is disturbed by phase and amplitude errors that are randomly distributed in the arrayed waveguides. This increases chromatic dispersion. Because the errors increase with decreasing channel separation, the chromatic dispersion increases similarly.

Another mode of the reduction of chromatic dispersion is utilizing of the dispersion compensated fibers.

\section{CROSSTALK}

When considering a signal out of a WDM demultiplexing port, there is always an amount of power, other than the useful one, belonging to other channels passing through the AWG device. This effect is generally referred as crosstalk, and it is usually classified as out-ofband or in-band crosstalk [8]. Crosstalk is caused due to imperfect construction of AWG. The other causes of crosstalk are: phase errors arising from variations in the arrayed waveguide width, thickness, material composition, and stress. The crosstalk influence of the adjacent channels each other are high, and non-adjacent channels are lower, but it increases with the number of the ports. If same wavelengths are used in the input of NxN AWG, the maximal admissible value of $\mathrm{N}$ severely limited (less than 20) [9]. It means that crosstalk can be reduced by using limited and odd numbers of the input ports of AWG for same wavelengths.
The crosstalk can be reduced by adjusting the phase delays in the individual arrayed waveguides, also by reducing of the numbers of the input ports, which use same wavelengths.

\section{POLARIZATION DEPENDENT DISPERSION}

If the array waveguides are equal or fundamental $\mathrm{TE}$ (electrical field vector parallel to the wafer plane) - and TM electrical field vector perpendicular to the wafer plane) -modes have constant propagation the AWG exhibits identical spectral response for any polarization.

The propagation constants of TE-and TM-modes have any difference, results in a shift $\Delta$ of the spectral response for TE- or TM-polarized modes, which is called polarization dispersion.

Several methods have been presented to reduce this polarization dependence, such as making the Free Spectral Range equal the difference between the phase change between TE and TM polarized modes, hence overlapping the TE/TM spots [10], or using a polarization converting lambda half-plate half way along the AWs [11], causing both polarizations to undergo the same phase change. Stress birefringence in the waveguides is treated to some extent by coating the waveguides with a stress inducing film or, as covered in [12], in the case of silicon on Silica waveguides, stress-relieving grooves can be cut either side of the waveguide.

\section{POLARIZATION ROTATION}

AWG device is bent from his nature. In any curved device the light energy is transferred from one polarization to the other and this process is called Polarization Rotation. There are the polarization conversion devices where Polarization Rotation is used in [13], [14]. This field of AWG was investigated very little.

\section{PASSBAND SHAPE}

A passband with a sharp top allows very little errors in AWG wavelength tolerance. The ideal shape for the passband of an AWG is to have a flat top [15]. There are several methods of flatting of the passband of AWG, such as creating a spot that has a broad flat centre with steep cut-off at either side, what has two types: Multi-Mode Interference Devices (MMIs) [16] and y-junctions or a parabolic tapered horn [17]; broadening the capture width of the receiver waveguide by using wide, multimode waveguides; spatial filtering [18]; using of sub-parabolic chirp [19].

In [20] was considered the method of flattening the spectral response of the $\mathrm{AWG}$, where the spectral response is manipulated by altering the respective lengths of each arm of the AWG and their positions at the edge of FPRs.

\section{INSERTION LOSS AND NON-UNIFORMITY}

There are various loss mechanisms in AWG. The most valuable of them occurs in the junctions between FPR (coupler) and arrayed waveguides. Junction losses for practical devices are between 1 and $2 \mathrm{~dB}$ per junction (i. e. between $2-4 \mathrm{~dB}$ for the total device). Propagation loss in 
the AWG and coupling losses due to a mismatch between the imaged field and the receiver waveguide mode are usually much smaller. In this case the other channels (waveguides) of AWG have more losses than the central channel (waveguide).

Insertion loss can be reduced by: 1) adding some waveguides where the gap between the waveguides is found to be large; 2) properly choosing of the parameters of the attenuation constant and the initial step in the initial design of AWG [20].

A silica-based AWG with particularly low insertion loss of only $0.75 \mathrm{~dB}$ has been achieved using a structure with vertically tapered waveguides in the intersection region between the arrayed waveguides and the slab which reduces the transition loss at that interface. In addition, a spot-size converter was incorporated into the AWG in order to reduce the fiber-to waveguide coupling loss [21].

Another highly effective method to reduce the insertion loss of an AWG, which is based on the same idea of tapering, has been patented by Lucent: A segmented transition region is inserted between the slab and the arrayed waveguides, which comprises a number of paths intersecting the waveguide array and exhibiting progressively decreasing width as they depart further away from the slab [22].

The non-uniformity of an AWG can be reduced by increasing the FSR, however, at the expense of a larger device size.

\section{PASSBAND POSITION}

The phase errors can occur due to the design errors or fabrication imperfections. The phase errors are the causes of displacement the focal point away from expected position so affecting the passband position. Temperature correcting allows shifting the position of the focal point and so compensates displacement [23]. The separate heaters can be used in the case when the phase error is random for each waveguide, causing the spot to defocus. But this method increases the energy consumption of the device and requires additional control circuitry, what makes manufacture expensive.

\section{BLOCKING}

One of the problems concern to AWG is blocking. This process is observed when it is used as a switching fabric. If in the given state it is not possible to connect an idle input terminal to an idle output terminal, but there are other states in which this connection is possible, than this state is called a blocking state. This issue can be reduced by the implementation of Tunable and Fixed wavelength converters (TWC and FWC, respectively), optical buffering and deflection routing.

\section{PHASE ERRORS}

One source of crosstalk is phase error. It can be compensated by trimming all waveguides in the AWG individually, but it is laborious and time consuming [24], [25]. Ideally, the AWG routes each wavelength to a different output port without causing any channel crosstalk at the other AWG output ports. However, real
AWGs suffer from leakage due to optical path phase errors [26]. The ways of the correction of the phase errors are:

1) The phase correction can be achieved by ultraviolet-induced refractive index changes in the glass. All the waveguides are exposed at the same time by using a metal mask with different opening lengths over each waveguide that are proportional to the phase errors to be compensated.

2) In AWG filter, waveguides with $750 \times 200-\mathrm{nm}^{2}$ cores are used to reduce phase errors due to the variation of core width [27].

3) An alternative method is to use additional bandpass filters at the output ports of a conventional AWG.

\section{CONCLUSIONS}

AWG is one of important element (device) for all optical networks. The effort of all scientists of the world is directed on improving the characteristics of existing AWG, such as lower insertion loss, improved crosstalk, higher channel resolution, higher total channel count, lower chromatic dispersion, amplitude and phase errors and so on. Despite many research have been carried out, still there are many problems which require some solutions. For instant, the polarization rotation is not researched enough. It is necessary to research of fully occurrence of Amplitude and Phase errors, the optimal ways of reducing of chromatic dispersion in AWG. And elimination of polarization and temperature sensitivity are actually nowadays.

\section{ACKNOWLEDGMENTS}

This work was supported by projects KEGA No: 006TUKE-4/2014 (25\%) and APVV-0025-12: Mitigation of Stochastic Effect in High-Bitrate All Optical Networks (75\%).

\section{REFERENCES}

[1] KARTALOPOULOS, S.V.: DWDM Networks, Devices, and Technology, IEEE Press, New York, 2001.

[2] RAMASWAMI, R. - SIVARAJAN, K.- SASAKI, G.: Optical Networks: A Practical Perspective, Elsevier, Amsterdam, 2009.

[3] ZYSKIND, J. - SRIVASTAVA, A.: Opticaly Amplified WDM Networks, Elsevier, Amsterdam, 2011.

[4] TAKAHASHI, H. - SUZUKI, S. - KATO, K. NISHI, I.: Arrayed-waveguide grating for wavelength division multi/demultiplexer with nanometer resolution, Electron.Lett.26, 1990, 87-88.

[5] SMIT, M.K. - VAN DAM, C.: PHASAR-based WDM devices: Principles, design and applications, IEEE Journ. of Selected Topics on Quantum Electronics, Vol. 2, No. 2, June 1996, 236-250. 
[6] DOERR, C.R. - JOYNER, C.H.: Double Chirping of the Waveguide Grating Router, IEEE Photon Technol. Lett. Vol.9, No. 6, June, 1997.

[7] ABD EL - NASER A. Mohammed, Ahmed Nabih Zaki Rashed - Abd El - Fattah A. Saad: Applications of Arrayed Waveguide Grating (AWG) in Passive Optical Networks, International Journal of Future Generation Communication and Networking, Vol. 2, No. 2, June, 2009, 25-36.

[8] RAMASWAMI, R. - SIVARAJAN, K.N.: Optical Networks, A Practical Perspective. San Fransisco, CA: Morgan Kaufmann, 1998.

[9] GAUDINO, R. - CASTILlO, G.G. - NERI, F. FINOCHIETTO, J.: Simple optical fabrics for scalable terabit packet switches, IEEE ICC, May, 2008, 5331-5337.

[10] SPIEKMAN, L.H. - AMERSFOORT, M.R.: Design and Realization of polarization independent phased array wavelength demultiplexers using different array orders for TE and TM, J Lightwave Technol., Vol. 14, 1996, 991-995.

[11] TAKAHASHI, H. - HIBINO, Y. - NISHI, I.: Polarization-insensitive arrayed waveguide grating wavelength multiplexer on silicon", Opt. Lett., Vol. 17, No. 7, 1992, 499-501.

[12] NADLER, C.K. - et al.: Polarization Insensitive, Low-Loss, Low-Crosstalk Wavelength Multiplexer Modules, J. Selected Topics in Quantum Electron., Vol. 5, No. 5, 1999, 1407 - 1412.

[13] VAN DAM, C. - et al.: Novel compact polarization converters based on ultra- short bents, IEEE Photon. Technol. Lett., Vol.8, 1996, 1346-1348.

[14] LUI, W.W. - HIRONO, T. - YOKOYAMA, K.Y. HUANG, W.P.: Polarization Rotation in Semiconductor Bending Waveguides: a coupledmode theory formulation, J. Lightwave Technol., Vol. 16, No. 5, 1998, 929 -936.

[15] MCGREER, K.A.: Arrayed Waveguide Gratings for Wavelength Routing, University of Manitoba and TRLabs, IEEE commications Magazine, December, 1998.

[16] MUNOZ, P. - PASTOR, D. - CAPMANY, J.: Analysis and design of arrayed waveguide gratings with MMI couplers, Optics Express, Vol. 9, No. 7, 2001.

[17] OKAMOTO, K. - SUGITA, A.: Flat Spectral response Arrayed Waveguide Grating multiplexer with parabolic waveguide horns, Electron. Lett., Vol. 32, 1996, 1661-1662.

[18] DRAGONE, C. - STRASSER, T. - BOGERT, G.A. - STULZ, L.W. - CHOU, P.: Waveguide grating router with maximally flat channel passband produced by spatial filtering, Electron. Letters, Vol. 33, No. 15, July, 1997, 1312-1314.

[19] PARKER, M.C. - WALKER, S,D..: Design of Arrayed Waveguide Gratings Using Hybrid Fourier-
Fresnel Transfom Techniques, IEEE J. Select. Areas Quantum. Electron., Vol. 5, No. 5, September/October, 1999, 1379-1384.

[20] KAMALAKIS, T. - SPHICOPOULOS, T.: An Efficient Technique for the Design of an ArrayedWaveguide Grating with Flat Spectral Response, J. Lightwave Technol., Vol. 19, No. 11, 2001, 17161725.

[21] SUGITA, A. - KANEKO, A. - OKAMOTO, K. ITOH, M. - HIMENO, A. - OHMORI, Y.: Very low insertion loss arrayed-waveguide grating with vertically tapered waveguides, J. Lightwave Technol., Vol.12, 2000, 1180-1182.

[22] LI, Y.P.: US Patent No. 5,745,618, 1998.

[23] YAMADA, H. - TAKADA, K. - MITACHI, S,: Crosstalk Reduction in a $10 \mathrm{GHz}$ Spacing ArrayedWaveguide Grating by Phase-Error Compensation, $J$. of Lightwave Technology, Vol. 16, No. 3, March, 1998.

[24] TAKADA, K. - YAMADA, H. - INOUE, Y.: Optical low coherence method for characterizing silica-based arrayed-waveguide grating multiplexers, J. Lightwave Technol., Vol. 14, 1996, 1677-1689.

[25] PAWLOWSKI, E. - KUHLOW, B. PRZYREMBEL, G. - WAMUTH, C.: Arrayedwaveguide grating demultiplexer with variable center frequency and transmission characteristic, Proc. 9th Eur. Conf. on Integr. Opt. (ECIO'99) Torino, Italy, 1999, 207-210.

[26] TAKADA, K. - YAMADA, H. - INOUE, Y.: Origin of channel crosstalk in $100 \mathrm{GHz}$-spaced silica-based arrayed-waveguide grating multiplexer. Electronics Letters, Vol.3, No. 14, July, 1995, 1176-1177.

[27] BOGAERTS, W. - DUMON, P. - VAN THOURHOUT, D. - TAILLAERT, D. - JAENEN, P. - WOUTERS, J. - BECKX, S. - WIAUX, V. BAETS, R.: Compact wavelength-selective functions in silicon-on-insulator photonic wires. IEEE J. Sel. Top. Quantum. Electron., Vol. 12, 2006, 1394-1401.

Received April 11, 2014 , accepted September 19, 2014

\section{BIOGRAPHIES}

Abdulazizjon ABDULLAEV received B.Sc. and M.Sc. degrees in telecommunication from Tashkent University of Information Technologies, Tashkent, Uzbekistan, in 2000 and 2002, respectively. Since 2001 until 2009 he worked in the enterprise companies of the field of telecommunication. He works as a senior teacher of the Fergana Branch of Tashkent University of Information Technologies since 2009. His research interests are optical networks, Arrayed Waveguide Grating (AWG), early diagnostics of optical networks, optical transport and access networks. 
Ján TURÁN (Dr.h.c., Prof., RNDr., Ing. DrSc.) in radioelectronics from University of Technology, received Ing. (MSc.) degree in physical engineering Košice, Slovakia, in 1983, and 1992, respectively. with honours from the Czech Technical University, Since March 1979, he has been at the University of Prague, Czech Republic, in 1974, and RNDr. (MSc.) Technology, Košice as Professor for electronics and degree in experimental physics with honours from telecommunication technology. His research Charles University, Prague, Czech Republic, in interests include digital signal processing and fiber 1980. He received a CSc. (PhD.) and DrSc. degrees optics, communication and sensing. 